Cuadernos de Filología Italiana

ISSN: $1133-9527$

https://dx.doi.org/10.5209/cfit.61766

\title{
Una Lupa tra lupe. Riflessioni filologiche e traduttive nella combinazione linguistica italiano-spagnolo-francese
}

Giorgia Marangon ${ }^{1}$

Recibido: 03 de octubre de 2018 / Modificado: 14 de febrero de 2019 / Aceptado: 02 de julio de 2019

Riassunto. Questa indagine centra la sua attenzione nell'analisi filologica e dei risultati traduttivi in spagnolo e francese di alcuni frammenti della novella La Lupa di Giovanni Verga, raccolta nell'opera Vita dei campi (1880). Abbiamo scelto, per la sua comparazione, la traduzione spagnola del granadino José Abad Baena, Cavalleria rusticana y otros cuentos sicilianos $\left(2011^{2}\right)$, e quella francese, della svizzera Béatrice Haldas, Cavalleria rusticana et autre nouvelles siciliennes (2013). I risultati pratici ottenuti sono utili per uno studio approfondito della traduzione dei fenomeni di variazione linguistica tra lingue affini.

Parole chiave: filologia, traduzione, lingue affini, Verga, La lupa.

\section{[en] A she- wolf among wolves. Philological and traductological reflections in the Italian-Spanish and French language combination}

\begin{abstract}
This paper attempts to provide a philological analysis which focuses on the translation results in Spanish and French of the Italian story La Lupa by Giovanni Verga, collected in the work Vida dei campi (1880). For their comparison we have selected the translation into Spanish by José Abad Baena from Granada, Cavalleria rusticana y otros cuentos sicilianos (2011), and the French translation by the Swiss Béatrice Haldas, Cavalleria rusticana et autres nouvelles siciliennes (2013). The practical results obtained are useful in our view for an in-depth study of the translation of the phenomena of linguistic variation between related languages.
\end{abstract}

Keywords: Philology, translation, cognate languages, Verga, she-wolf.

Sommario: 1. Introduzione 2. Stato dell'arte 3. Una Lupa tra lupe 4. Analisi filologica e dei risultati traduttivi in spagnolo e francese 5. Osservazioni conclusive.

Come citare: Marangon, Giorgia (2019): «Una Lupa tra lupe. Riflessioni filologiche e traduttive nella combinazione linguistica italiano-spagnolo-francese», Cuadernos de Filología Italiana, 26, pp. 221232.

\footnotetext{
1 Universidad de Córdoba. Departamento de Ciencias del Lenguaje, Plaza del Cardenal Salazar s/n, ES-14071 Córdoba.

1r1marmg@uco.es
} 


\section{Introduzione}

«Nihil est dictum, quod non sit dictum prius». (Hieronymus

Stridonius, I, 9: 233)

Porsi criticamente di fronte alla Lupa di Verga mette lo studioso in una situazione di sconcerto. Il testo, pur considerato solo in quanto racconto, ha conosciuto una mole di studi sia diretti che trasversali, e sotto diverse angolazioni, sì che resta oggettivamente difficile affrontare una novella così compatta nella sua essenziale brevità con la speranza di sviscerare del nuovo (Paccagnini 2000: 31).

Questo studio, che si apre con la citazione del professor Ermanno Paccagnini dell'Università Cattolica del Sacro Cuore, ha come fil rouge l'analisi filologica e dei risultati traduttivi in lingua spagnola e francese della novella La Lupa, la seconda che compone l'opera Vita dei campi (1880) dello scrittore siciliano Giovanni Verga. Le traduzioni prese in esame per il confronto traduttivo sono La Loba del granadino José Abad Baena, inclusa in Cavalleria rusticana y otros cuentos sicilianos (2011) e La Louve della svizzera Béatrice Haldas, raccolta nell'opera Cavalleria rusticana et autres nouvelles siciliennes (2013). Allo stato attuale dell'indagine, queste traduzioni sono tra i lavori più recenti in ambito ispanofono e francofono. Quanto meno curioso è il calco del titolo delle raccolte, entrambi gli autori mantengono la prima parte Cavalleria rusticana in italiano permettendo al lettore di ritrovare, in assenza di quella musicalità siciliana tanto cara a Verga, l'essenziale della visione verghiana, tra lucidità ed emozione. La traduzione spagnola contiene dieci novelle corredate da note pubblicate alla fine del libro, mentre quella francese riunisce più raccolte: La vie aux champs, Nouvelles paysannes e Vagabondages.

\section{Stato dell'arte}

Il critico italiano Felice Cameroni aveva fatto leggere i primi testi di Verga ad uno scrittore della corrente del naturalismo svizzero, Edouard Rod. Quest'ultimo, arrivato a Parigi per incontrare un gruppo di scrittori naturalisti al seguito di Zola, si appassionò all'opera verghiana e ne divenne il suo primo traduttore in Francia. A testimoniarne il lavoro è la fitta corrispondeza epistolare tra i due ${ }^{2}$. «Ho uno stanzino grande come la mano da mettere a vostra disposizione, una vera cella da frate [...]» (Verga 1954: 7), così nel settembre del 1882, Giovanni Verga invitava Édouard Rod che veniva ad incontrarlo a Milano, nel suo appartamento di corso Venezia. La loro amicizia, cominciata intorno ad un progetto di traduzione dei Malavoglia, era destinata ad essere soprattutto epistolare. Queste lettere sono la testimonianza diretta ed esplicita di come Verga e i suoi scritti siano arrivati al pubblico francese. In un'intervista al giornale italiano la Repubblica (2009), Dominique Budor, docente di letteratura italiana presso l'Università Sorbonne Nouvelle Paris 3, spiega:

[...] l'idea che la fortuna di Verga in Francia sia un dato di fatto, qualcosa di consustanziale alla stessa cultura e letteratura francese, alla luce dei rapporti che lega-

Si tratta di 153 lettere conservate nella Biblioteca Cantonale e Universitaria di Losanna. 
rono lo scrittore siciliano alle cose e alle vicende d' oltralpe è, in realtà, croce e delizia dello stesso autore. Nel senso che, dalle nostre parti, è ancora particolarmente in voga la propensione a leggere la produzione del grande siciliano come una derivazione dal naturalismo.

\section{E continua:}

Ma se è vero che in Francia ancora perdura uno spirito sciovinista, in forza del quale Verga sarebbe un prodotto di Zola, va detto anche che lo scrittore di Vizzini fu subito tradotto, alla fine del diciannovesimo secolo, ad opera di Éduard Rod: Sì, fu lui il suo primo traduttore in Francia: era uno svizzero arrivato a Parigi ed entrato in contatto con Verga perché faceva parte del gruppo degli scrittori naturalisti. Lui si appassionò all'opera di Verga, ma bisogna dire che conosceva maluccio l'italiano, quindi nei suoi lavori rimangono irrisolti alcuni problemi legati alla interpretazione del testo. Da allora, la situazione è di certo migliorata, ma attualmente non si registra un particolare fervore relativamente alla traduzione in francese dell'opera verghiana. Questo aspetto dipende probabilmente da scelte opportunistiche legate al mercato editoriale, forse si pensa che Verga venderebbe meno degli scrittori siciliani contemporanei, abbondantemente tradotti dalle nostre parti.

Bisogna aspettare il 1976 per avere una traduzione all'altezza dell'autore tradotto: Cavalleria rusticana et autres nouvelles siciliennes. Questa raccolta, firmata dalla traduttrice Béatrice Haldas e da Georges Haldas, che ne firma la prefazione, è ripresa, titolo compreso, e pubblicata in edizione moderna dalla casa editrice Belle Lettres nel 2013. Nella prefazione, Georges Haldas ci presenta le grandi tappe della vita dello scrittore siciliano. Il famoso traduttore, poeta e scrittore ginevrino s'interroga sulle ragioni dell'oblio che pervade l'opera di Verga in Francia: « On dirait que sur l'œuvre du prosateur sicilien, sur sa vie même et sa mémoire, un interdit pèse» (Haldas 2013:10). Afferma, inoltre: «Quiconque va en Sicile, devrait avoir lu quelques pages de Verga. Quiconque a lu Verga, devrait aller en Sicile» (Haldas 2013:9).

Situazione molto simile in ambito ispanofono, dove, seppur presenti traduzioni di prestigio come quelle di Cipriano Rivas Cherif(1920) e Guillermo Fernández (2007), tra le altre, e le più recenti di Hugo Bachelli (2008) e José Abad Baena $\left(2011^{2}\right)^{3}$, non si riesce a trasmettere quella fisionomia caratteristica siciliana tanto ricercata e cara a Verga. Consapevole di queste difficoltà, Verga si mette a disposizione del traduttore per aiutarlo a capire, a sentire la realtà contadina e l'amina dei suoi personaggi.

Milano, 4 dicembre, 1881 [...] Son certo che dalle sue mani escirà un lavoro perfetto per quanto le consente la diversità dell'indole delle due lingue, e la difficoltà enorme che Ella dovrà incontrare a rendere in francese uno stile che ho cercato di ridurre non solo personalmente ma possibilimente immedesimato all'argomento che si svolge in ambiente e fra personaggi assai diversi dal comune. Onde agevolare il suo compito io mi metto a sua disposizione per aiutarla in quelle parti della traduzione dove il carat-

3 Grazie all'aggiornata banca dati della pagina web spagnola "Base de datos de libros publicados en España" e al catalogo delle traduzioni spagnole di opere italiane fino al 1939 del "Proyecto Boscán", abbiamo circoscritto il materiale bibliografico alle traduzioni spagnole sopra citate. 
tere e l'indole dello stile siciliano sarebbero, non solo per lei straniero, ma anche per un italiano, di difficoltà insormontabile. [...] La prego soltanto di lasciare il testo nella sua integrità. I tagli che ella vorrebbe fare non solo fanno sanguinare il mio cuore d'autore, ma parmi che nuocerebbero assai al libro. Sento che non ho scritto nei Malavoglia né un rigo, né una parola di superfluo, e faccio appello a tutta la sua buona volontà per accettarlo tale e quale è (Verga 1954: 38-39).

Il professor Giorgio Longo (2008: 138), ricostruendo dai carteggi gli scambi tra gli autori, ripercorre le tracce dei rapporti e dei confronti tra Verga e Rod.

I suoi carteggi con Verga costituiscono un prezioso strumento non solo come materiale biografico-memoriale, o per lo studio della diffusione degli scrittori italiani in Francia, ma soprattutto per la conoscenza critico-teorica della letteratura italiana del periodo. L'approccio di Rod costituisce infatti un curioso esempio di conoscenza critica dall'interno, che ci fornisce nel contempo una serie di documenti a volte di importanza fondamentale.

A guidare il nostro lavoro traduttivo-comparativo nella combinazione linguistica italiano-spagnolo-francese sono proprio le parole di Giovanni Verga, dello scrittore siciliano che nel binomio lingua-dialetto riflette i colori, i profumi di una terra e, nella terra, le miserie dei suoi abitanti, $\mathrm{i}$ vinti.

\section{Una $L_{u p a^{4}}$ tra lupe}

La gnà Pina, la Lupa del titolo, è nel racconto l'incarnazione della sessualità istintiva e animale. Ė l'immagine della femminilità primitiva, inquietante e senza controllo, caratteristiche tipiche dei molti personaggi femminili ritratti dallo scrittore. Ha tratti somatici da strega, da donna incantatrice, sirena ${ }^{5}$, ma le sue armi non sono la grazia e la cultura, ma un insieme di forze occulte, le più segrete e poderose della natura. La Lupa incarna il binomio donna/bestia, donna/demonio in cui prendono forma e corpo le forze incoscienti e incontrollabili dell'istinto, quell'istinto selvaggio, animale, fuori dai canoni e dall'accettazione sociale. La leggenda avvolge il personaggio in un'aura di solitaria grandiosità; la Lupa rappresenta il "diverso", lo "sconosciuto" e per questo è temuta, emarginata ed esclusa dalla società. Non ci si può proteggere dalla sua presenza, dalla sua forza, dall'irruenza della sua fisicità.

La novella è suddivisibile in quattro principali sequenze narrative: il ritratto iniziale della Lupa, l'innamoramento, l'amore incestuoso tra genero e suocera, la morte/sacrificio che ristabilisce l'ordine delle cose.

Per lo studio dei risultati traduttivi in spagnolo e francese prenderemo in esame solo la protagonista, la sua minuziosa descrizione fisica e intima, personale, il suo ritratto disegnato con maestria dal Verga, che si riflette come una costante nelle quat-

4 Pubblica la novella nella Rivista nuova di Scienze, Lettere e Arti e poi la inserisce nella raccolta Vita dei campi, del 1880: sedici anni dopo ne fa una trasposizione teatrale di successo a Torino (con accenti più realistici), cosa che verrà ripetuta nel 1953 da Lattuada (che sposta l'ambientazione dalla Sicilia alla Lucania), nel 1966 da Zeffirelli, sino al film di G. Lavia trent'anni dopo.

5 Le Sirene, divinità di antichissima origine orientale, nel mito greco rappresentano i pericoli derivanti dalla bellezza ingannatrice e dall'abbandono del proprio autocontrollo. 
tro sequenze narrative e che prende forma e si costruisce in un crescendo di passione, delirio, irreligiosità e trasgressione.

Abbiamo analizzato, seguendo la teoria traduttologica di Jean Delisle (1993), che si applica per l'analisi degli errori commessi nella traduzione, quattro untità testuali.

\section{Analisi filologica e dei risultati traduttivi in spagnolo e francese}

La novella, che prende spunto da un fatto di cronaca, l'omicidio di una contadina a mano del genero come conseguenza di una incestuosa passione amorosa ${ }^{6}$, si apre con la descrizione della protagonista introdotta da un tempo verbale che i grammatici definiscono «imperfetto onirico» (Serianni 1988: 396), ossia da resoconto.

Era alta, magra; aveva soltanto un seno fermo e vigoroso da bruna e pure non era più giovane; era pallida come se avesse sempre addosso la malaria, e su quel pallore due occhi grandi così, e delle labbra fresche e rosse, che vi mangiavano (Verga 1996: 197).

L'assenza del soggetto e la sequenza imperfetto + aggettivo conferiscono alla protagonista una natura eroica; la magrezza ${ }^{7}$, che non è sinonimo di fragilità, risulta essere la sua forza, il suo vigore, «e pure non era più giovane», ma aveva un «seno fermo e vigoroso da bruna». Nel gioco delle contrapposizioni ritroviamo una donna «pallida», ma con «gli occhi grandi così», messi in rilievo dal sintagma nominale e con un'accezione favolistica espressa dall'aggettivo «grandi». Il motivo degli occhi ritorna per nove volte nella novella. Gli occhi come specchio dell'anima, un'anima turbata dal desiderio che tutto travolge e trasforma. Quegli occhi non mentono e

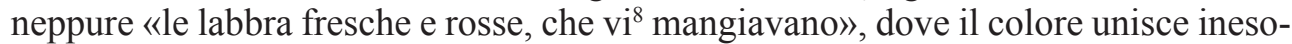
rabilmente il fuoco della passione e il sangue della morte, mentre l'insaziabile voracità espressa dal verbo mangiare, che ritorna nel testo, alimenta quella passione cieca e bruciante. L'immagine della Lupa appare al lettore in tutta la sua drammatica realtà; Verga la situa, fin dall'inizio, in una realtà testuale colma di riferimenti all'universo stregonico-demoniaco; la Lupa pecca, ma il suo peccare, come sottolineava Luigi Russo (1959), porta in se stesso la sua pena, il suo castigo.

Questa specie di terrore religioso della passione incestuosa, che è nella protagonista, si diffonde anche negli altri, ché istintivamente la loro voce pettegola s'abbassa di tono come davanti ad uno spirito maligno. [...] Certo il terrore religioso della passione è un elemento della primitività dei personaggi verghiani, ma è anche un

$6 \quad$ Lo stesso Luigi Capuana (1880 [1988: 69-77]) ce ne dà testimonianza nella celebre recensione alla novella: «I personaggi di questi racconti, la più parte, hanno esistito realmente, e l'autore non ha fatto che degli studi dal vero. Quella Lupa io l'ho conosciuta. Tre mesi fa, tra le colline di S. Margherita, su quel di Mineo. [...] Si vedeva ritta, innanzi il pagliaio, all'ombra dei rami d'un ulivo in maniche di camicia, col fazzoletto rosso sulla testa, spiando le viottole, pallida come avesse sempre addosso la malaria [...]. Più spesso si vedeva andare di qua e di là per la campagna sola come una cagnaccia, con quell'andare randagio e sospetto della lupa, come il Verga l'ha dipinta superbamente».

$7 \quad$ Verga pare ben cosciente della ricchezza constante delle sfaccettature allegoriche della Lupa. Non possiamo non ricordare il verso 49 (Inferno I) di Dante: «Ed una lupa, che di tutte brame / sembiava carca ne la sua magrezza / e molte genti fè già viver grame».

$8 \quad$ L'inserimento del pronome di seconda persona $v i$ introduce il punto di vista popolare. 
segno della profonda religiosità che c'è nel popolo siciliano o meglio nel Verga. Religiosità sui generis che difficilmente si può costringere in una confessione cioè in una dottrina (Russo 1959: 100, 103).

Ma vediamo come i traduttori si approcciano a un testo di questo tipo e di queste carratteristiche linguistiche e culturali.

\begin{tabular}{|c|c|}
\hline José Abad Baena (2011) & Béatrice Haldas (2013) \\
\hline $\begin{array}{l}\text { Era alta, delgada, y no tenía más que un } \\
\text { pecho firme y vigoroso de mujer morena. } N i \\
\text { siquiera era joven. Era pálida como si sufriera } \\
\text { permanentemente la malaria y, sobre esa } \\
\text { palidez, tenía dos ojos así de grandes, y labios } \\
\text { frescos y rojos que te comían. (37) }\end{array}$ & $\begin{array}{l}\text { Elle était grande, magre, mais avec des seins } \\
\text { fermes et vigoureux de brune, - et cependant } \\
\text { elle n'était plus jeune ; elle était pâle, comme } \\
\text { si elle avait toujours la malaria, et dans cette } \\
\text { pâleur, deux yeux grands comme ça, et les } \\
\text { lèvres fraîches qui vous mangeaient.(61) }\end{array}$ \\
\hline
\end{tabular}

La volontà di rimanere fedeli al testo origine $(\mathrm{TO})$ è chiara fin dalla traduzione del titolo: La Loba in spagnolo e La Louve in francese, mantenendo la forte carica metaforica che Verga attribuisce al soprannome dato alla protagonista. «La traduzione è la riproduzione nella lingua di arrivo (...) dell'equivalente naturale più prossimo al messaggio comunicato nella lingua di partenza» (Nida 1964: 121). Se entriamo nel dettaglio delle traduzioni, possiamo vedere come Béatrice Haldas preferisce l'aggettivo grande a haute, forse perché nel primo è insito il secondo e dà all'immagine della protagonista una certa imponenza fisica. Abad Baena traduce letteralmente. In entrambe le traduzioni si perde il rinforzo dell'avverbio «soltanto», molto difficile da rendere, che spezza il tessuto narrativo della novella; l'avverbio mette in evidenza il seno della donna, un seno «fermo e vigoroso» che simboleggia la femminilità, la fertilità e la prosperità. È un seno da bruna, da donna del sud. Abad Baena usa pecho potendo calcare seno, mantenuto dalla Haldas al plurale, seins. Per continuare, la forma analitica «e pure», è un elemento lessicale unico, la cui traduzione corretta in spagnolo è aunque o sin embargo e non ni siquiera e, in francese è cependant come ritroviamo nel testo della svizzera. Nella seconda parte del frammento, troviamo in entrambi i testi tradotti l'omissione del termine «addosso», riferito alla malaria. «Avere addosso la malaria» ha una carica semantica molto più forte di «essere malato di malaria» o «avere la malaria»; quell' «addosso» incarna l'aggressività della malattia e le conseguenze di averla contratta, tra cui quel «pallore» che le faceva risaltare ancor di più gli «occhi grandi così». L'espressione sufrir la malaria usata da Abad Baena non è corretta in spagnolo, crediamo che possa essere stato influenzato dall'italiano «soffrire di malaria». Un'altra duplice omissione, questa volta più grave, è quella commessa dalla traduttrice; omette sia il colore rosso delle

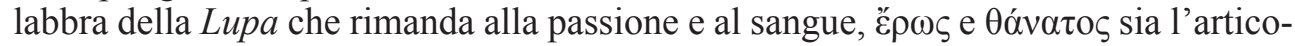
lo partitivo «delle» riferito a «labbra», che sostituisce con il determinativo les. Mantiene invece quel «vi», seguito dal verbo «mangiare» che lo spagnolo traduce con te, facendoci perdere il punto di vista popolare. Il partitivo è omesso anche da Abad Baena che, a differenza della svizzera, non sostituisce ma aggiunge un verbo che nel testo originale non è presente: il verbo tener che introduce «occhi» e «labbra».

Dopo una prima breve descrizione della Lupa data nell'incipit della novella, vediamo come vengono messe in evidenza le caratteristiche ricorrenti della stessa che 
si muove in una dimensione ferina/umana e stregonico/leggendaria. Nel seguente esempio si conosce il soprannome che i compaesani attribuiscono a comare Pina: Lupa, che rimanda direttamente al modo di dire siciliano aviri la lupa che significa 'divorare', 'avere gran fame' (Pasqualino 1789: 62), inteso nella novella nel senso metaforico di appettito carnale, rafforzato dall'incidentale esplicativa «non era sazia giammai - di nulla». Anche questa frase presenta la non facile traduzione di un avverbio, in questo caso si tratta di giammai che è la forma rafforzata aulica di mai:

«Al villaggio la chiamavano la Lupa perché non era sazia giammai - di nulla». (Verga 1996: 197)

\begin{tabular}{|l|l|}
\hline \multicolumn{1}{|c|}{ José Abad Baena (2011) } & \multicolumn{1}{|c|}{ Béatrice Haldas (2013) } \\
\hline $\begin{array}{l}\text { En el pueblo la llamaban la Loba porque no se } \\
\text { saciaba nunca, de nada. (37) }\end{array}$ & $\begin{array}{l}\text { Au village, on l'appelait la Louve, car elle n'était } \\
\text { jamais repue - de rien. (61) }\end{array}$ \\
\hline
\end{tabular}

La traduttrice, a differenza di Abad Baena, mantiene il soprannome di comare Pina in corsivo come nel testo origine (TO) e traduce letteralmente «Al villaggio», mentre il termine spagnolo pueblo, seppur corretto, crediamo che dovrebbe essere sostituito dal termine aldea per le caratteristiche geografiche e di giurisdizione che lo accomunano con il termine d'origine. Crediamo, inoltre, che il giammai italiano abbia una connotazione più forte del nunca o del jamais; nonostante questo, la sospensione della virgola e la negazione che segue in entrambi i testi meta (TM), ne migliorano la traduzione.

Continuando con la descrizione della Lupa e sottolineando le sue caratteristiche di donna/bestia e donna/demonio, ci soffermiamo a riflettere sul seguente passaggio verghiano che introduce l'irreligiosità e la trasgressione della protagonista:

Le donne si facevano la croce quando la vedevano passare, sola come una cagnaccia, con quell'andare radagio e sospettoso della lupa affamata; ella si spolpava i loro figliuoli e i loro mariti in un batter d'occhio, con le sue labbra rosse, e se li tirava dietro alla gonnella solamente a guardarli con quegli occhi da satanasso, fossero stati davanti all'altare di Santa Agrippina (Verga 1996: 197).

\begin{tabular}{|c|c|}
\hline José Abad Baena (2011) & Béatrice Haldas (2013) \\
\hline $\begin{array}{l}\text { Las mujeres se hacian el signo de la cruz } \\
\text { cuando la veían pasar, sola como una perra, } \\
\text { con ese paso errabundo y suspicaz de loba } \\
\text { hambrienta. Ella devoraba a sus hijos y maridos } \\
\text { en un abrir y cerrar de ojos, con sus labios } \\
\text { rojos, y los ponía tras sus faldas solo con } \\
\text { echarles encima esos ojos de Satanás suyos, así } \\
\text { estuviesen ante el altar de Santa Agripina. (37) }\end{array}$ & $\begin{array}{l}\text { Les femmes se signaient, quand elles la voyaient } \\
\text { passer, seule, comme une mauvaise chienne, } \\
\text { avec ce pas errant et soupçonneux de luove } \\
\text { affamée; elle ne faisait qu'une bouchée de leurs } \\
\text { fils et de leurs maris, avec ses lèvres rouges, et } \\
\text { rien qu'à les regarder de ses yeux de possédée, } \\
\text { elle les traînait derrière ses jupes, eussent-ils eté } \\
\text { devant l'autel de sainte Agrippine. (61) }\end{array}$ \\
\hline
\end{tabular}

In questo frammento, risalta l'intervento ironico di Verga che chiama in causa Dio, gli angeli, la Madonna, Gesù in un solo nome, quasi a sottolineare la sfida sovrumana perpetrata e vinta dalla Lupa. 
Richiama l'attenzione del lettore il calco semantico usato da Abad Baena nella traduzione dell'espressione «farsi il segno della croce»; il termine corretto è hacer(se) la señal de la cruz o santiguarse. Corretta è invece la traduzione francese.

Il termine «cagnaccia» viene mantenuto nella sua forma al femminile da entrambi i traduttori; ci sembra corretta l'aggiunta fatta dalla Haldas dell'aggenttivo mauvaise per rendere ancora di più la connotazione negativa del verghiano «cagnaccia» (cagna + suffisso peggiorativo -accia).

Un altro punto che è importante chiarire è quello relativo al verbo «spolpare». Non possiamo tralasciare l'origine dialettale del verbo: spruppari in siciliano (Pasqualino 1895: 134), la cui etimologia ci riporta all'attuale spolpare: togliere la polpa dall'osso, ma che, nell'uso popolare indica, ancor oggi, l'azione di mangiare con voracità. I segni distintivi del personaggio sono, infatti, «le labbra fresche e rosse e gli occhi grandi così», o «neri come il carbone», tutte caratteristiche fisiche affini rispetto al mangiare e con allusività erotica, rimarcata dalla metafora dello spolpare, al "mangiare qualcuno con gli occhi". La scelta del traduttore granadino è azzeccata, anche se l'omissione del secondo «loro» riferito a «mariti» indebolisce il verso; ci lascia invece perplessa la scelta della Haldas. Riformula l'espressione azzerando le connotazioni aggressive, erotiche che il verbo "spolpare» e il corrispondente spagnolo devorar trasmettono.

Il verbo sintagmatico «tirare dietro» è reso in modo letterale dalla traduttrice svizzera, non è compreso invece dallo spagnolo, che lo traduce erroneamente. Un sinonimo di «tirare dietro» è «attirare», ossia atraer, llevar tras, ir tras, arrastrar tras in spagnolo e non poner tras.

Nell'ultima parte di questo frammento, Haldas traduce quel «fossero stati davanti all'altare di Santa Agrippina» mantenendosi fedele al TO procurando al lettore meta le stesse sensazioni del lettore origine. Nell'espressione verghiana è implicito un "come se" che Abad Baena traduce con un avverbio dimostrativo así, che dà origine a un controsenso dovuto a una errata interpretazione, che tradisce il pensiero dell'autore.

Un altro elemento caratterizzante della protagonista, oltre al soprannome datole dai compaesani, è il prefisso gnà che precede il nome. Gnà è il soprannome che nei dialetti siciliano e calabrese significa 'signora'. Il termine ha origine spagnola, è una adattazione grafica dello spagnolo $\tilde{n} a$, abbreviazione di doña, dueña ed è un prefisso che si usa per indicare le donne di paese, le popolane di condizione sociale bassa. José Abad Baena ha scelto l'opzione naturalizzante di questo termine e ha tradotto gnà come doña. Questa scelta causa nel TM una perdita d'informazione del TO; doña, infatti, si usa per riferirsi a una signora di ceto sociale alto. Anticamente veniva utilizzato per differenziare il plebeo dal nobile o il creolo dalla persona comune. Cipriano Rivas Cherif, nella sua La Loba, contenuta nella raccolta Vida en los campos (1920), usa il termine señá che, anche foneticamente, ci riporta allo gnà di Giovanni Verga. Un'altra possibilità potrebbe essere l'uso del termine comadre con l'accezione di vicina e/o amica. Béatrice Haldas, in tutta la sua raccolta, mantiene saggiamente il prefisso in italiano e fornisce al lettore una breve spiegazione in $\mathrm{Ned}$ $d a$ (Haldas 2013: 27-28).

La novella termina con la Lupa in primo piano; non si tratta di una descrizione fisica come in apertura, ma intimistica, caratteriale. Il cerchio si chiude e l'ordine delle cose viene ristabilito grazie alla morte sacrificale della protagonista a mano del genero. L'uccisione della Lupa viene solo preannunciata dai gesti, dalle parole, dal 
progressivo chiudersi del cerchio, inteso come spazio scenico. La scena finale si articola come in una tragedia classica nel momento in cui si compie un destino fatale, predeterminato, insito nel personaggio. La morte, infatti, rappresenta il momento di massima affermazione della Lupa che è disposta a sacrificare la propria vita pur di rimanere coerente ai propri sentimenti, passioni e pulsioni. È consapevole che il suo sacrificio redimerà il suo Nanni e ripristinerà l'ordine al villaggio.

Il punto di rottura è quando Nanni sopravvive, grazie all'intervento del conforto religioso, all'incidente con il mulo per poi ricadere immediatamente nella colpa, nel peccato diventando spergiuro non solo davanti alla moglie e figlia della Lupa, ma anche e soprattutto davanti a Dio. Ed ecco l'espressione-sentenza che il coro narratore enuncia: «E meglio sarebbe stato per lui che fosse morto in quel giorno, prima che il diavolo tornasse a tentarlo e a ficcarglisi nell'anima e nel corpo quando fu guarito» (Verga 1996: 201) Un'esecuzione annunciata, biblica, pronunciata su Nanni, alla quale si può sottrarre solo eliminando la causa del suo peccare, e questo la Lupa lo sa.

La Lupa lo vide venire, pallido e stralunato, colla scure che luccicava al sole, e non si arretrò di un sol passo, non chinò gli occhi, seguitò ad andargli incontro, con le mani piene di manipoli di papaveri rossi, e mangiandoselo con gli occhi neri. Ah! malanno all'anima vostra! balbettò Nanni (Verga 1996: 201).

\begin{tabular}{|l|l|}
\hline José Abad Baena (2011) & Béatrice Haldas (2013) \\
\hline $\begin{array}{l}\text { La Loba lo vio acercarse, pálido, fuera de sí, con } \\
\text { el hacha reluciendo al sol, y sin dar un solo paso } \\
\text { atrás ni bajar los ojos, fue a su encuentro, las } \\
\text { manos llenas de amapolas rojas, comiéndoselo } \\
\begin{array}{l}\text { con los ojos negros. -iAh! ¡Maldita sea su alma! vit venir, pâle et hagard, avec se } \\
\text {-balbuceó Nanni. (42) }\end{array}\end{array}$ & $\begin{array}{l}\text { hache qui brillait au soleil; et elle ne recula } \\
\text { pas d'un pouce, ne baissa pasa les yeux, elle } \\
\text { continua de marcher à sa recontre, le bras } \\
\text { chargé de pavots rouge, et le dévoran de ses } \\
\text { yeux noirs. - Ah ! malheur à votre âme ! } \\
\text { balbutia Nanni. (65) }\end{array}$ \\
\hline
\end{tabular}

La traduzione francese è letterale e trasmette al lettore il pathos dell'ultima scena, quel binomio amore-morte che è il fil rouge della novella. Lo spagnolo omette la doppia negazione presente nel TO, una negazione di gran forza espressiva non si arretrò e non chinò; il solo ni non è sufficiente. Un'altra omissione è quella della congiunzione $e$ davanti a mangiandoselo; Abad Baena, dopo la virgola, traduce direttamente il verbo comiéndoselo; anche manipoli è omesso da entrambi i traduttori come pure il corsivo con cui Verga scrive il nome della protagonista. Omissioni, queste, che indeboliscono e impoveriscono i TM.

La Lupa è il motore di una novella che conquista il lettore fin dai primi gesti, citando Capuana (1988: 69-77):

La Lupa è forse la più bella cosa che il Verga abbia mai scritto; senza forse, è la miglior novella di questa Vita dei campi. La forma è scultoria, di una semplicità meravigliosa. Qua e là sembra una traduzione di qualche leggenda popolare, con quel ritorno di immagini e di parole di cui l'autore si è stupendamente servito. 


\section{Osservazioni conclusive}

Per concludere l'analisi e fare il punto della questione, riassumiamo gli errori di traduzione più frequenti rivelateci dal confronto delle traduzioni menzionate della novella, seguendo la classificazione di Delisle. L'analisi filologica e l'accurata ricerca dei riscontri traduttivi ci hanno portato ad analizzare un numero determinato di elementi linguistici che hanno dato luogo, nella maggior parte dei casi, a errori di traduzione. Ne abbiamo estrapolati una ventina e, nel riscontro traduttologico abbiamo classificato gli errori più ricorrenti per numero e ripetizione. Nella traduzione spagnola abbiamo riscontrato nove errori di perdita della funzione poetica, sette gravi omissioni, due calchi rilevanti e due errori di controsenso e di ipertraduzione; in quella francese, tre errori di perdita della funzione poetica e cinque omissioni.

Il maggior numero di errori si riassumono nella perdita del tono generale del testo tradotto, del messaggio, delle sfumature, della resa di parole chiave della scrittura verghiana e nelle numerose e gravi omissioni.

La traduzione di questi testi non può limitarsi a un fatto semplicemente linguistico ma dev'essere operazione che coinvolge un ambito molto più vasto, interazione tra culture; è l'unione che Meschonnic (1973: 305-323) chiama «langue-culture». Secondo il filosofo e linguista tedesco, Wilhelm von Humboldt, ogni lingua è causa e riflesso della Weltanschaung di un popolo, che è alla base della sua cultura. Riferendosi alla traduzione, Humboldt sostiene che la sua finalità sia proprio quella di cogliere e trasmettere quella visione del mondo propria di un popolo e lo spirito della cultura presso la quale il testo originale è stato composto. Evidenti sono le similitudini con il pensiero di Giovanni Verga:

[...] So bene la grande difficoltà che vi è a tradurre questi schizzi che hanno già una fisionomia tutta loro anche nell'italiano. Il mio è un tentativo nuovo sin qui da noi, e tuttora molto discusso, di rendere nettamente la fisionomia caratteristica di quei racconti siciliani nell'italiano; lasciando più che potevo l'impronta loro propia, e il loro accento di verità. Per Lei, francese, le difficoltà saranno maggiori, e faccio assegnamento non solo sulla sua buonissima conoscenza della nostra lingua, ma su tutto il suo talento di artista e la sua penetrante intuizione di critico. Le mando come prova un tentativo [...], ha voluto fare della Lupa, la più accentuata delle novelle di Vita dei campi. Essa non le sarà forse inutile per avere un'idea di quel che riuscirebbe il tentativo che le propongo lasciando più che possiblile allo scritto nella traduzione francese la sua fisionomia caratteristica siciliana, come io ho cercato di renderla nell'italiano [...] (Verga 1954: 28-30).

Crediamo che le traduzioni prese in esame, seppur migliorabili, affrontino con rigore e sensibilità, i problemi traduttivi summenzionati ma non è sufficiente. Seppure la ricerca della fedeltà sia al primo posto per entrambi i traduttori, manca nelle loro opere un connubio filologo/traduttore. Alla presenza di un testo dove lingua e dialetto sono inscindibili, il binomio filologo/traduttore deve intervenire nel testo con acutezza e sensibilità per sentire, vedere e parlare come i protagonisti, come il proprio autore, e poter così dar forma a queste sensazioni nella traduzione. Solo così la nostra Lupa si mimitizzerà tra le lupe tradotte e arriverà al pubblico straniero con tutta la sua forza, irruenza, passione e trasgressione che la caratterizzano. 


\section{Riferimenti bibliografici}

Alighieri, Dante (2004): La Divina Commedia, a cura di Natalino Sapegno, Firenze, La Nuova Italia.

Capuana, Luigi (1880[1988]): «Rassegna letteraria Vita dei campi di G. Verga», Corriere della sera (20-21 settembre); poi in Studi di letteratura contemporanea (1882), seconda serie, Catania, Giannotta; ora, a cura di Paola Azzolini, Napoli, Liguori, pp. 69-77.

Delisle, Jean (1993): La traduction raisonnée. Manuel d'initiation à la traduction professionnelle de l'anglais vers le français. Ottawa, Presses de l'Université d'Ottawa.

Hieronimus Stridonius, Commentarius in Ecclesiasten, cap. 1, v. 9. [0347-0420].

Longo, Giorgio (2008): «Traduttori-imitatori: Pod, Eekhoud, Verga», Annali della Fondazione Verga, 17, pp. 137-152.

Meschonnic, Henri (1973[1981]): «Poétique de la traduction», Pour la poétique II, Paris, Gallimard. [Traduzione italiana di M. Corenna e D. D’Oria, «Proposizioni per una poetica della traduzione», Il lettore di provincia, 44, pp. 23-31].

Nida, Eugene (1964): Toward a Science of Translating, Leiden, Brill.

Paccagnini, Ermanno (2000): «Una Lupa tra lupe», Annali della Fondazione Verga, 17, pp. 31-85.

Pasqualino, Michele (1789): Vocabolario siciliano etimologico, italiano, e latino, Tomo III, Palermo, Reale stamperia.

Pasqualino, Michele (1895): Vocabolario siciliano etimologico, italiano, e latino, Tomo V, Palermo, Reale stamperia.

Russo, Luigi (1959): Verga novelliere e romanziere. Torino, Eri.

Serianni, Luigi (1988): Grammatica italiana. Italiano comune e lingua letteraria. Torino, Utet.

Verga, Giovanni (1954): Lettere al suo traduttore, a cura di Fredi Chiappelli, Firenze, Le Monnier.

Verga, Giovanni ( $\left.{ }^{5} 1996\right)$ : Giovanni Verga, Tutte le novelle, introduzione, testo e note di Carla Riccardi, Milano, Mondadori.

Verga, Giovanni (22011): Cavalleria rusticana y otros cuentos sicilianos, traduzione, prologo e note di José Abad Baena, Barcelona, Traspiés.

Verga, Giovanni (2013): Cavalleria rusticana et autres nouvelles siciliennes, traduzione di Béatrice Haldas, prologo di Georges Haldas, Parigi, Le Belles Lettres.

Verga, Giovanni (1976): Cavalleria rusticana et autres nouvelles siciliennes, traduzione di Béatrice Haldas, prologo di Georges Haldas, Parigi, Denoël.

Verga, Giovanni (1920): La vida en los campos. Novelas cortas, traduzione, prologo e note di Cipriano Rivas Cherif, Madrid, Calpe.

Verga, Giovanni (2007): Giovanni Verga, 4 cuentos veristas, traduzione, prologo e note di Guillermo Fernández, Ciudad de México, Universidad Autónoma Nacional de México.

Verga, Giovanni (2008): La Vida en el campo, traduzione, prologo e note di Hugo Bachelli, Cáceres, Periférica.

\section{Sitografia}

Base de datos de libros publicados en España (ISBN), http://www.mcu.es/webISBN/tituloSimpleDispatch.do;jsessionid=2F326074057E27DFD6DF956A823B563A 
Felita, Salvatore (2009): «Verga il re del verismo che consquistò i francese», http://ricerca. repubblica.it/repubblica/archivio/repubblica/2009/12/15/verga-il-re-del-verismo-checonquisto.html, [visto il 7/9/2018].

Proyecto Boscán, http://boscan.uv.es:591/CATALOGO/ FMPro 